\title{
Is the number of fast-food outlets in the neighbourhood related to screen-detected type 2 diabetes mellitus and associated risk factors?
}

\author{
Danielle H Bodicoat ${ }^{1}$, Patrice Carter ${ }^{1, *}$, Alexis Comber ${ }^{2}$, Charlotte Edwardson ${ }^{1}$, Laura J Gray ${ }^{3}$, \\ Sian Hill ${ }^{1}$, David Webb ${ }^{1}$, Thomas Yates ${ }^{1}$, Melanie J Davies ${ }^{1}$ and Kamlesh Khunti ${ }^{1}$ \\ 'University of Leicester, Diabetes Research Centre and Leicester Diabetes Centre, Leicester General Hospital, \\ Gwendolen Road, Leicester LE5 4PW, UK: ${ }^{2}$ University of Leicester, Department of Geography, Leicester, UK: \\ ${ }^{3}$ University of Leicester, Department of Health Sciences and Leicester Diabetes Centre, Leicester General Hospital, \\ Leicester, UK
}

Submitted 5 March 2014: Final revision received 13 August 2014: Accepted 17 September 2014: First published online 31 October 2014

\begin{abstract}
Objective: We investigated whether a higher number of fast-food outlets in an individual's home neighbourhood is associated with increased prevalence of type 2 diabetes mellitus and related risk factors, including obesity.

Design: Cross-sectional study.

Setting: Three UK-based diabetes screening studies (one general population, two high-risk populations) conducted between 2004 and 2011. The primary outcome was screen-detected type 2 diabetes. Secondary outcomes were risk factors for type 2 diabetes.

Subjects: In total 10461 participants (mean age 59 years; $53 \%$ male; $21 \%$ nonWhite ethnicity).

Results: There was a higher number of neighbourhood (500 $\mathrm{m}$ radius from home postcode) fast-food outlets among non-White ethnic groups $(P<0.001)$ and in socially deprived areas $(P<0 \cdot 001)$. After adjustment (social deprivation, urban/ rural, ethnicity, age, sex), more fast-food outlets was associated with significantly increased odds for diabetes (OR $=1 \cdot 02 ; 95 \%$ CI 1.00, 1.04) and obesity (OR = 1.02; $95 \%$ CI 1.00, 1.03). This suggests that for every additional two outlets per neighbourhood, we would expect one additional diabetes case, assuming a causal relationship between the fast-food outlets and diabetes.

Conclusions: These results suggest that increased exposure to fast-food outlets is associated with increased risk of type 2 diabetes and obesity, which has implications for diabetes prevention at a public health level and for those granting planning permission to new fast-food outlets.
\end{abstract}

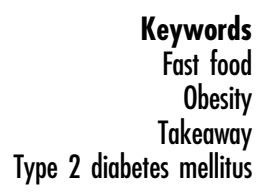

Type 2 diabetes mellitus is a growing epidemic, with estimates suggesting that worldwide prevalence will increase from 366 million in 2011 to 552 million in $2030^{(1)}$. Prevalence is closely associated with increasing obesity rates ${ }^{(2)}$ and is linked to environmental changes that have led to more sedentary lifestyles and poor-quality dietary intake ${ }^{(3)}$. Therefore, while interventions aimed at individuals changing behaviours can be successful ${ }^{(4)}$, population-level environmental changes are also needed if we are to curb the obesity epidemic and consequently the heavy burden of obesityrelated disease, such as type 2 diabetes ${ }^{(5)}$.

Consumption of fast food is a factor commonly linked with the obesity epidemic and there is some scientific evidence from adult populations to support this claim. Regular fast-food consumption has been linked to low adherence to dietary recommendations ${ }^{(6)}$. Greater energy density, high fat content, high levels of trans-fatty acids, high sodium content and larger portion sizes of fast food may all potentially contribute to overall poor diet quality $^{(6-9)}$. There is, however, limited evidence in adults at the population level to suggest that the number of fastfood outlets in an area is associated with obesity levels, with some ${ }^{(10)}$, but not all ${ }^{(11,12)}$ studies finding that more outlets were associated with increased obesity levels, possibly due to methodological weaknesses in some studies such as self-reported height and weight.

The existing research outlined above suggests that fast food might be a reasonable target for public health interventions aimed at reducing obesity and related conditions, including type 2 diabetes. There are however key gaps in 
the literature; namely, a lack of data exploring the association between fast food and type 2 diabetes, as well as a lack of data from Europe and in high-risk minority ethnic groups, such as South Asians ${ }^{(10-13)}$. The present analyses of over 10000 individuals from a multi-ethnic UK population are the first step towards addressing these gaps. We aimed to investigate the relationship between the number of fast-food outlets in an individual's neighbourhood and screendetected type 2 diabetes and associated risk factors.

\section{Experimental methods}

\section{Study population}

The present analyses used data from three studies (ADDITION-Leicester ${ }^{(14)}$, Let's Prevent Diabetes ${ }^{(15)}$ and Walking Away from Diabetes ${ }^{(16)}$ ), which were conducted by the same research group in Leicestershire, UK and used identical standard operating procedures. The studies were conducted according to the guidelines laid down in the Declaration of Helsinki and all procedures involving human participants were approved by the University Hospitals of Leicester and Leicestershire Primary Care Research Alliance (ADDITION-Leicester) and the Nottingham (Walking Away from Diabetes/Let's Prevent Diabetes) Research Ethics Committees. Written informed consent was obtained from all participants.

The studies have been described in detail elsewhere ${ }^{(14-16)}$. Briefly, they involved screening individuals for type 2 diabetes and then conducting randomised controlled trials for those found either to have type 2 diabetes (ADDITION-Leicester) or to be at high risk of developing it (Let's Prevent Diabetes and Walking Away from Diabetes). The data used for the present analyses were based on only the cross-sectional screening stage of each study. ADDITION-Leicester (2004-2009) was a population-based screening programme for people aged 40-75 years (White European) or 25-75 years (other ethnic groups). Let's Prevent Diabetes (2009-2011) screened individuals, at high risk of type 2 diabetes on a risk score, aged 40-75 years (White European) or 25-75 years (other ethnic groups). Walking Away from Diabetes (2010) also screened individuals at high risk of type 2 diabetes on a risk score, but adults aged 18-75 years were eligible. The studies recruited from primary care (response rate $=22 \%$ in all three studies ${ }^{(17)}$ ) and had similar exclusion criteria, which included a previous diagnosis of type 2 diabetes. All participants screened in these studies were potentially eligible for inclusion in the present analyses. Individuals were excluded if they had a missing or invalid postcode (zip code), as it was not possible to define a neighbourhood for these individuals. If an individual participated in more than one study then the most recent record was kept.

\section{Variables}

The biochemical, anthropometric and demographic variables used in the present analyses were measured during the screening visit ${ }^{(14-16)}$. The primary outcome variable was type 2 diabetes diagnosed using the WHO 2011 guidelines as fasting glucose $\geq 7 \cdot 0 \mathrm{mmol} / \mathrm{l}, 2 \mathrm{~h}$ glucose $\geq 11.1 \mathrm{mmol} / \mathrm{l}$ or glycated $\mathrm{Hb}$ (HbA1c) $\geq 6.5 \%$ (48 mmol/ $\mathrm{mol})^{(18)}$. Since people with known diabetes were excluded from screening, these individuals are those with undiagnosed type 2 diabetes, rather than all type 2 diabetes cases. Fasting blood samples for glucose and $\mathrm{HbA1c}$ were obtained following a minimum fast of $8 \mathrm{~h}$. An oral glucose tolerance test was then performed where participants consumed a standard measure of glucose and had another blood test $2 \mathrm{~h}$ later. Fasting glucose, $2 \mathrm{~h}$ glucose and HbA1c were also considered separately as secondary outcomes, as were several other variables. The presence of impaired glucose regulation, a high risk state for type 2 diabetes, was diagnosed as fasting glucose between 6.1 and $6.9 \mathrm{mmol} / \mathrm{l}$, $2 \mathrm{~h}$ glucose between 7.8 and $11.0 \mathrm{mmol} / \mathrm{l}$, or $\mathrm{HbA} 1 \mathrm{c}$ between 6.0 and $6.4 \%$ ( 42 and $46 \mathrm{mmol} / \mathrm{mol}$ ). BMI was calculated from height and weight which were measured by trained staff. Obesity was defined as BMI $\geq 30 \mathrm{~kg} / \mathrm{m}^{2}$ for all participants, except South Asians for whom it was defined as $\geq 27.5 \mathrm{~kg} / \mathrm{m}^{2(19)}$. Total, LDL and HDL cholesterol were also measured in the fasting blood sample, as was fasting insulin in Walking Away from Diabetes ( $n$ 654) and for a random subset of ADDITION-Leicester participants ( $n$ 905). These outcomes were chosen as they may mediate the association between fast-food consumption and type 2 diabetes, as well as being risk factors for other conditions.

The primary explanatory variable in the present analyses was the absolute number of fast-food outlets in the participant's neighbourhood. Neighbourhood was defined as the Euclidean distance within $500 \mathrm{~m}$ of the participant's home (identified through his/her postcode, which in the UK contains fifteen individual addresses on average). There is no standard definition of neighbourhood, therefore we chose to use a definition of $500 \mathrm{~m}$ as this distance is commonly used in physical activity studies, such as neighbourhood walkability studies ${ }^{(20-22)}$, and additionally considered the effect of this through sensitivity analyses. The locations of fast-food outlets were extracted from an online business listing (Thompson's directory) in January 2014 using the following search terms: 'fast food', 'fish and chips' and 'take away' to fit with the standard definition of fast food (i.e. hot food cooked on site that can be produced quickly and taken away). This means that the majority of outlets labelled as 'fast-food outlets' were self-defined when the outlets signed up for the business directory. Adding additional search terms, such as 'pizza', did not alter the number of outlets that were identified. The following confounding variables were also considered. Age, sex and ethnicity were all self-reported by the participant. Ethnicity was self-reported based on the British Census categories, but was grouped as White European (White British, White Irish, other White background), South Asian (Indian, Pakistani, Bangladeshi, other Asian background) and Other (Black Caribbean, Black African, other Black background, 
White and Asian, White and Black Caribbean, White and Black African, other mixed background, Chinese, other Chinese background, other background), due to a small number of participants in some ethnic groups. Index of Multiple Deprivation 2007 scores were used as a measure of social deprivation (http://data.gov.uk/dataset/index_of_ multiple_deprivation_imd_2007). These scores are a publicly available postcode measure, which are calculated using a variety of indicators including income, employment, education and living environment. A higher score indicates higher deprivation. An urban/rural indicator was obtained from the Office for National Statistics. This groups morphologies as urban (>10000 residents); town and fringe; or villages, hamlets and isolated dwellings. For these analyses, the two latter categories were grouped as 'rural'. Physical activity was measured using the short version of the International Physical Activity Questionnaire (IPAQ) and published standards were used to clean the data and produce estimates of the number of metabolic equivalents of task (MET) per day for total activity ${ }^{(23)}$.

\section{Statistical analysis}

The main purpose of the present analyses was to investigate whether the number of neighbourhood fast-food outlets was associated with screen-detected type 2 diabetes and other metabolic risk factors. Descriptive characteristics by study and for the overall population are presented. Continuous variables are summarised as mean and standard deviation and categorical variables as number and percentage. The mean (SD) number of fast-food outlets was summarised overall and within subgroups of patient characteristics. ANOVA was used to test for a difference in the number of outlets within subgroups. Generalised estimating equation models were fitted separately for each primary (type 2 diabetes) and intermediate (impaired glucose regulation, obesity, BMI, waist circumference, fasting glucose, $2 \mathrm{~h}$ glucose, HbA1c, total cholesterol, LDL cholesterol, HDL cholesterol, fasting insulin) outcome. For each of these outcomes, an unadjusted and adjusted model was fitted. Adjusted models included the following confounders: social deprivation (continuous), urban/rural indicator (continuous), ethnicity (White European, South Asian, Other), age (continuous) and sex (male, female). The generalised estimating equation models had the number of fast-food outlets as the explanatory variable, a term to allow for clustering within postcodes, exchangeable correlation structures and robust standard errors. The distribution and link function were binomial and logit for binary outcome variables, and Gaussian and identity for continuous outcome variables. Physical activity (total $\mathrm{MET} / \mathrm{d}$ ) was also considered as a confounder; however, it did not change the results and so it was not included in the presented models because this variable was available for only $82 \%$ of the sample and including it substantially reduced the sample size unnecessarily. Sensitivity analyses were repeated for the adjusted analyses using different distances for the definition of neighbourhood and using the density (number of outlets per 200 residents, where neighbourhood population size was obtained from 2011 Census data) of neighbourhood fast-food outlets in a $500 \mathrm{~m}$ radius as the explanatory variable instead of the absolute number of outlets. Interaction terms between the number of fast-food outlets and ethnicity, urban/rural indicator and social deprivation score were fitted in turn. All analyses were performed in the Stata statistical software package version 13 and $P$ values are two-sided. A $P$ value of less than 0.05 was considered statistically significant for main effects and $P$ value of less than $0 \cdot 1$ for interactions. Missing data were not imputed.

\section{Results}

ADDITION-Leicester screened 6749 participants, of whom 300 were excluded because they had a missing postcode and six because they had an invalid one, leaving 6443 eligible participants. Let's Prevent Diabetes screened 3450 participants, of whom eighteen had an invalid postcode, thus 3432 participants were eligible. Walking Away from Diabetes screened 833 participants, of whom three had an invalid postcode, thus 830 participants were eligible. There were 244 people who participated in more than one of the studies, so the present analyses included a total of 10461 participants, whose characteristics are shown in Table 1.

In summary, the participants were aged $59 \cdot 0$ (SD 10.4) years old on average and $53 \%$ were male. ADDITIONLeicester participants tended to be slightly younger and were more likely to be female compared with the other two studies. This is because ADDITION-Leicester was a general population screening study, whereas the other two studies screened high-risk individuals. There were a mean of $2 \cdot 1$ ( $\mathrm{sD} 3 \cdot 7$; range $=0-36$ ) fast-food outlets in participants' neighbourhoods (Table 2). The number of neighbourhood fast-food outlets was significantly higher for South Asians than White Europeans $(P<0 \cdot 001)$, in urban areas compared with rural areas $(P<0.001)$ and near those with high compared with low social deprivation $(P<0.001)$.

Table 3 shows the model estimates for the association between the number of fast-food outlets and each outcome. In unadjusted analyses, the number of fast-food outlets was positively associated with screen-detected type 2 diabetes (OR $=1.05 ; 95 \%$ CI 1.04, 1.07; $P<0.001$ ), 2 h glucose (unstandardised regression coefficient, $b=0.02$; $95 \%$ CI 0.00, 0.03; $P=0 \cdot 01)$, HbA1c ( $b=0 \cdot 01 ; 95 \%$ CI 0.00, $0 \cdot 01 ; P<0.001)$ and fasting insulin $(b=0.13 ; 95 \%$ CI 0.04 , $0.22 ; P<0.01)$. After adjustment for social deprivation score, urban/rural indicator, ethnicity, age and sex, the positive association with type 2 diabetes was attenuated but remained significant $(\mathrm{OR}=1 \cdot 02$; $95 \%$ CI $1.00,1 \cdot 04$; $P=0.02$ ), while the associations with each of $2 \mathrm{~h}$ glucose, 
Table 1 Descriptive characteristics of the study population: multi-ethnic men and women attending three UK-based diabetes screening studies (one general population, two high-risk populations) conducted between 2004 and 2011

\begin{tabular}{|c|c|c|c|c|c|c|c|c|}
\hline \multirow[b]{2}{*}{ Variable } & \multicolumn{2}{|c|}{$\begin{array}{l}\text { ADDITION-Leicester } \\
(n \text { 6200) }\end{array}$} & \multicolumn{2}{|c|}{$\begin{array}{l}\text { Let's Prevent Diabetes } \\
\qquad(n \text { 3431) }\end{array}$} & \multicolumn{2}{|c|}{$\begin{array}{l}\text { Walking Away from } \\
\text { Diabetes ( } n \text { 830) }\end{array}$} & \multicolumn{2}{|c|}{$\begin{array}{c}\text { All } \\
(n 10461)\end{array}$} \\
\hline & Mean & SD & Mean & SD & Mean & SD & Mean & SD \\
\hline Age (years) & $56 \cdot 19$ & $10 \cdot 78$ & $63 \cdot 17$ & $8 \cdot 11$ & $63 \cdot 11$ & $8 \cdot 18$ & 59.03 & $10 \cdot 37$ \\
\hline Social deprivation score & $19 \cdot 74$ & $14 \cdot 11$ & $17 \cdot 32$ & 14.99 & $20 \cdot 22$ & $16 \cdot 31$ & 18.99 & 14.63 \\
\hline BMI $\left(\mathrm{kg} / \mathrm{m}^{2}\right)$ & 28.00 & 5.03 & 32.45 & $5 \cdot 70$ & 32.44 & 5.62 & 29.85 & $5 \cdot 75$ \\
\hline Waist circumference (cm) & $93 \cdot 74$ & $13 \cdot 21$ & $107 \cdot 50$ & 39.90 & $101 \cdot 80$ & $12 \cdot 38$ & 98.99 & $26 \cdot 24$ \\
\hline Fasting glucose $(\mathrm{mmol} / \mathrm{l})$ & $5 \cdot 19$ & 0.91 & $5 \cdot 33$ & 0.84 & $5 \cdot 32$ & 0.79 & $5 \cdot 25$ & 0.88 \\
\hline $2 \mathrm{~h}$ glucose $(\mathrm{mmol} / \mathrm{l})$ & 6.01 & 2.43 & $6 \cdot 62$ & 2.50 & 6.49 & 2.42 & $6 \cdot 25$ & 2.47 \\
\hline $\mathrm{HbA} 1 \mathrm{c}(\%)$ & $5 \cdot 70$ & 0.61 & 5.94 & 0.52 & 5.92 & 0.58 & 5.79 & 0.59 \\
\hline Total cholesterol (mmol/l) & 5.54 & 1.08 & 5.09 & 1.03 & 5.09 & 1.07 & $5 \cdot 35$ & 1.09 \\
\hline LDL cholesterol (mmol/l) & 3.53 & 0.93 & 3.03 & 0.89 & 3.07 & 0.90 & 3.33 & 0.94 \\
\hline HDL cholesterol (mmol/li) & 1.37 & 0.38 & 1.39 & 0.44 & 1.39 & 0.37 & 1.38 & 0.40 \\
\hline \multirow[t]{2}{*}{ Fasting insulin $(\mu \mathrm{lU} / \mathrm{ml})$} & $10 \cdot 04$ & $8 \cdot 61$ & & & $10 \cdot 65$ & $7 \cdot 34$ & $10 \cdot 30$ & $8 \cdot 10$ \\
\hline & \multicolumn{2}{|c|}{$\%$} & \multicolumn{2}{|c|}{$\%$} & \multicolumn{2}{|c|}{$\%$} & \multicolumn{2}{|c|}{$\%$} \\
\hline Female & \multicolumn{2}{|c|}{$53 \cdot 1$} & \multicolumn{2}{|c|}{$39 \cdot 1$} & \multicolumn{2}{|c|}{$36 \cdot 6$} & \multicolumn{2}{|c|}{$47 \cdot 2$} \\
\hline White European & \multicolumn{2}{|c|}{$74 \cdot 0$} & \multicolumn{2}{|c|}{$86 \cdot 7$} & \multicolumn{2}{|c|}{88.4} & \multicolumn{2}{|c|}{$79 \cdot 4$} \\
\hline South Asian & \multicolumn{2}{|c|}{$23 \cdot 5$} & \multicolumn{2}{|c|}{$10 \cdot 7$} & \multicolumn{2}{|c|}{$8 \cdot 1$} & \multicolumn{2}{|c|}{$18 \cdot 0$} \\
\hline Other ethnicity* & \multicolumn{2}{|c|}{$2 \cdot 6$} & \multicolumn{2}{|c|}{$2 \cdot 6$} & \multicolumn{2}{|c|}{$3 \cdot 5$} & \multicolumn{2}{|c|}{$2 \cdot 7$} \\
\hline Rural location & \multirow{2}{*}{\multicolumn{2}{|c|}{$\begin{array}{r}11.7 \\
6.2\end{array}$}} & \multicolumn{2}{|c|}{24.5} & \multicolumn{2}{|c|}{17.5} & \multicolumn{2}{|c|}{$16 \cdot 3$} \\
\hline Screen-detected type 2 diabetes & & & \multirow{2}{*}{\multicolumn{2}{|c|}{$10 \cdot 9$}} & \multicolumn{2}{|c|}{9.4} & \multicolumn{2}{|c|}{$8 \cdot 0$} \\
\hline Impaired glucose regulation & \multicolumn{2}{|c|}{$25 \cdot 3$} & & & & & & \\
\hline Obese & & & & & & & & \\
\hline
\end{tabular}

$\mathrm{HbA1c}$, glycated $\mathrm{Hb}$.

Data are presented as mean and standard deviation or as percentage.

Missing values: social deprivation score, $n 6$; BMl/obesity, $n$ 208; waist circumference, $n$ 205; fasting glucose, $n$ 33; 2 h glucose, $n$ 81; total cholesterol, $n$ 108; LDL cholesterol, $n$ 467; HDL cholesterol, $n$ 139; fasting insulin, $n$ 8902; ethnicity, $n$ 190; urban/rural indicator, $n$ 6; type 2 diabetes, $n$ 13; impaired glucose regulation, $n 13$; other variables, $n 0$.

*The 'Other' ethnic group comprised $78 \%$ of individuals of Black ethnicity, $18 \%$ of individuals of mixed ethnicity and $4 \%$ of individuals who identified themselves as of another ethnic origin.

Table 2 Number of neighbourhood fast-food restaurants by participant characteristics among multi-ethnic men and women attending three UK-based diabetes screening studies (one general population, two high-risk populations) conducted between 2004 and 2011

\begin{tabular}{|c|c|c|c|c|}
\hline \multirow[b]{2}{*}{ Variable } & \multirow[b]{2}{*}{ Category } & \multicolumn{2}{|c|}{$\begin{array}{l}\text { Number of fast-food outlets within } 500 \mathrm{~m} \\
\text { of home postcode }\end{array}$} & \multirow[b]{2}{*}{$P$ value } \\
\hline & & Mean & SD & \\
\hline All & & 2.06 & 3.73 & \\
\hline \multirow[t]{3}{*}{ Age } & $<55$ years & $2 \cdot 61$ & $4 \cdot 27$ & \\
\hline & $55-64$ years & 1.95 & 3.64 & \\
\hline & $\geq 65$ years & 1.69 & $3 \cdot 22$ & $<0.001$ \\
\hline \multirow[t]{2}{*}{ Sex } & Men & 2.01 & 3.68 & \\
\hline & Women & $2 \cdot 12$ & $3 \cdot 78$ & 0.107 \\
\hline \multirow[t]{3}{*}{ Ethnicity } & White European & 1.53 & $3 \cdot 17$ & \\
\hline & South Asian & 3.96 & 4.73 & \\
\hline & Other & 4.57 & $5 \cdot 33$ & $<0.001$ \\
\hline \multirow[t]{2}{*}{ Urban/rural indicator } & Urban & 2.36 & 3.98 & \\
\hline & Rural & 0.53 & 1.06 & $<0.001$ \\
\hline \multirow[t]{2}{*}{ Social deprivation } & Low & 0.91 & 1.89 & \\
\hline & High & 3.53 & 4.83 & $<0.001$ \\
\hline
\end{tabular}

HbA1c and fasting insulin were no longer significant. Conversely, the positive associations with obesity, BMI and fasting glucose strengthened and became significant $(P<0.01$ for both obesity and BMI) or borderline significant ( $P=0.06$ for fasting glucose). There was no significant interaction on type 2 diabetes between outlets and ethnicity $(P=0 \cdot 21)$, outlets and urban/rural location $(P=0.98)$ or outlets and social deprivation score $(P=0.93)$.

Sensitivity analyses for different definitions of neighbourhood showed similar results (Supplemental Table 1, see online supplementary material). Interestingly, when neighbourhood was defined to cover a smaller region $(100 \mathrm{~m}$ or $250 \mathrm{~m})$, the relationship between the number of fast-food outlets and type 2 diabetes was weak but there were significant positive associations with adiposity measures. Conversely, for areas of neighbourhood covering larger regions $(500 \mathrm{~m}, 750 \mathrm{~m}$ or $1000 \mathrm{~m})$, the number of fast-food outlets was positively associated with type 2 diabetes, obesity and fasting glucose; these associations were small but significant. Sensitivity analyses using the 
Table 3 Association between the number of neighbourhood fast-food outlets and diabetes-related outcomes among multi-ethnic men and women attending three UK-based diabetes screening studies (one general population, two high-risk populations) conducted between 2004 and 2011

\begin{tabular}{|c|c|c|c|c|}
\hline \multirow[b]{2}{*}{ Outcome } & \multicolumn{2}{|c|}{ Unadjusted } & \multicolumn{2}{|c|}{ Adjusted* } \\
\hline & OR & $95 \% \mathrm{Cl}$ & OR & $95 \% \mathrm{Cl}$ \\
\hline Screen-detected type 2 diabetes & 1.05 & $1.04,1.07$ & 1.02 & $1.00,1.04$ \\
\hline Impaired glucose regulation & 1.00 & $0.99,1.01$ & 1.00 & $0.98,1.01$ \\
\hline \multirow[t]{2}{*}{ Obese } & 1.03 & $1.01,1.04$ & 1.02 & $1.01,1.03$ \\
\hline & $b$ & $95 \% \mathrm{Cl}$ & $b$ & $95 \% \mathrm{Cl}$ \\
\hline BMI $\left(\mathrm{kg} / \mathrm{m}^{2}\right)$ & 0.02 & $-0.02,0.05$ & 0.04 & $0.00,0.08$ \\
\hline Waist circumference $(\mathrm{cm})$ & -0.04 & $-0.19,0.11$ & $0 \cdot 10$ & $-0.04,0.25$ \\
\hline Fasting glucose $(\mathrm{mmol} / \mathrm{l})$ & 0.01 & $-0.00,0.01$ & 0.01 & $-0.00,0.01$ \\
\hline $2 \mathrm{~h}$ glucose $(\mathrm{mmol} / \mathrm{l})$ & 0.02 & $0.00,0.03$ & 0.00 & $-0.01,0.02$ \\
\hline $\mathrm{HbA} 1 \mathrm{c}(\%)$ & 0.01 & $0.00,0.01$ & 0.00 & $-0.00,0.01$ \\
\hline Total cholesterol (mmol/l) & -0.02 & $-0.02,-0.01$ & -0.01 & $-0.01,-0.00$ \\
\hline LDL cholesterol (mmol/l) & -0.01 & $-0.02,-0.01$ & -0.01 & $-0.01,-0.00$ \\
\hline HDL cholesterol (mmol//I) & -0.01 & $-0.01,-0.00$ & 0.00 & $-0.00,0.00$ \\
\hline Fasting insulin $(\mu \mathrm{lU} / \mathrm{ml}) \dagger$ & 0.13 & $0.04,0.22$ & 0.00 & $-0.11,0.12$ \\
\hline
\end{tabular}

$\mathrm{HbA1c}$, glycated $\mathrm{Hb} ; b$, unstandardised regression coefficient.

${ }^{*}$ Adjusted for social deprivation score, urban/rural indicator, ethnicity, age and sex.

†Only available for 1559 participants (905 from ADDITION-Leicester and 654 from Walking Away from Diabetes).

density, instead of the absolute number, of fast-food outlets demonstrated a similar pattern of results to the main analyses, although there were some differences in terms of which results were significant (Supplemental Table 2, see online supplementary material).

\section{Discussion}

In a multi-ethnic region of the UK, individuals had on average two fast-food outlets within $500 \mathrm{~m}$ of their home. This number differed substantially by key demographics, including ethnicity; people of non-White ethnicity had more than twice the number of fast-food outlets in their neighbourhood compared with White Europeans. We found that the number of fast-food outlets in a person's neighbourhood was associated with an increased risk of screen-detected type 2 diabetes and obesity, after adjustment for confounders. Associations with other intermediate outcomes were weak and generally non-significant after adjustment for confounders. The exceptions were BMI and fasting glucose, which displayed weak positive associations with the number of fast-food outlets.

This work has several notable strengths; namely, it is the first study, to our knowledge, to look at the association between the number of neighbourhood fast-food outlets and type 2 diabetes, it included a large sample size from a multi-ethnic population, gold-standard measures were used to obtain the outcomes, results were found to be reasonably robust in sensitivity analyses and due to the detailed data that were collected we were able to look at potential moderators of the relationships identified. Nevertheless, these results should be interpreted with consideration of the study's limitations. First, these are cross-sectional analyses, as with much of the literature on this topic; thus it is not possible for us to infer a causal effect. Although plausible causal mechanisms exist as discussed below, it is possible that demand precedes supply and that there are more fastfood outlets in the neighbourhood as that is the type of food that the residents want. Second, the number of the fast-food outlets was measured in 2014, while other variables were measured earlier, and only a sample of fast-food outlets was included in these analyses, due to time and cost constraints. The associations demonstrated are therefore likely to be attenuated and the relationship between fast-food outlets and obesity-related outcomes might in fact be stronger than observed.

The results of the present study add to the limited evidence that currently exists regarding fast-food outlets, obesity and type 2 diabetes in adults. The majority of previous studies conducted have taken place in the USA ${ }^{(24)}$, thus we provide novel data from a large multi-ethnic population within the UK, which increases the generalisability of our findings. Previous studies have demonstrated that increased exposure to fast-food outlets is inversely associated with healthy lifestyle score, adherence to dietary recommendations and overall diet quality, which are risk factors for type 2 diabetes $^{(6,7)}$. In comparison, a major strength of our study was that it looked directly at the relationship between fastfood outlets and type 2 diabetes. However, we did not differentiate between different types of fast-food outlet and so this is an area for potential future work. This would allow variations and nuances in the relationship between fast-food location/distance and incidence of type 2 diabetes to be identified, related to the nutritional level of the fast-food outlet. Moreover, future work or similar work in other countries could also include convenience stores, as they tend to be a common source of junk foods and sugared drinks.

The results support data from the Coronary Artery Risk Development in Young Adults (CARDIA) study, which 
found that increased fast-food consumption was associated with clinically relevant changes in CVD and type 2 diabetes risk factors including increased weight ${ }^{(8)}$. They also support the results of Smith et al. who found that regular fast-food consumption was associated with increased abdominal obesity ${ }^{(6)}$, and of Maddock who found that as the square miles per fast-food restaurant decreased (i.e. the density of restaurants increased), obesity prevalence measured at the state level increased ${ }^{(10)}$. The observed association between the number of fastfood outlets with obesity and type 2 diabetes does not come as a surprise; fast food is high in total fat, trans-fatty acids and sodium, portion sizes have increased twoto fivefold over the last 50 years $^{(9)}$ and a single fast-food meal provides approximately $5860 \mathrm{~kJ}(1400 \mathrm{kcal})^{(25)}$. The energy density, defined as the energy content per unit weight of foods, meals or diets ${ }^{(26)}$, of fast food may also be important, since individuals consume a relatively constant weight of food and therefore consumption of foods with high energy density may lead to a passive increase in energy intake ${ }^{(25)}$. Experiments have also shown that energy-dense diets challenge the innate ability to maintain energy homeostasis ${ }^{(26)}$. Furthermore, fast-food outlets often provide sugar-rich drinks. Carbonated drinks appear to bypass satiety mechanisms and the energy provided by them is not compensated for during meals ${ }^{(9)}$. It is also plausible that the observed associations are due to confounding. It has been shown that unhealthy behaviours (i.e. smoking, excessive alcohol use, poor diet and low levels of physical activity) cluster together ${ }^{(27)}$. In particular, poor diet and low physical activity tend to occur together, thus the observed association between fast-food outlets and obesity-related outcomes in the present study might also reflect known associations between obesity and lack of physical activity. However, this is unlikely since adjusting for physical activity did not alter the findings.

Other key findings of our study included observing very few significant associations between the number of neighbourhood fast-food outlets and diabetes risk factors. This contrasts with the CARDIA study which observed increases in homeostasis model assessment of insulin resistance, waist circumference and TAG, and reduced HDL cholesterol, with increased fast-food consumption ${ }^{(8)}$. These different findings may be because we measured the availability of fast food and the CARDIA study measured consumption $^{(8)}$. Our data also demonstrated a greater number of fast-food outlets in non-White than in White neighbourhoods as reported in studies conducted in the $\mathrm{USA}^{(7,24)}$. This is particularly relevant in the UK South Asian population who are known to be at increased risk of developing type 2 diabetes compared with their White European counterparts ${ }^{(22)}$, and suggests that environmental differences between ethnic groups might be contributing towards this problem ${ }^{(28)}$. Finally, we found that the association between the number of fast-food outlets and the various outcomes that we considered was somewhat dependent on the definition of neighbourhood that was used. We chose to define neighbourhood as the Euclidean distance within $500 \mathrm{~m}$ of the participant's home, since a standard definition of neighbourhood is not available but this is commonly used in the physical activity literature ${ }^{(20-22)}$. Sensitivity analyses using different distances in the definition suggested that when neighbourhood was defined to cover a smaller region there were positive associations with adiposity measures, whereas for larger regions there were positive associations with type 2 diabetes, obesity and fasting glucose. This might be a chance finding, or it could reflect that some definitions are more prone to confounding than others.

We estimated that for each additional fast-food outlet, there was a $2 \%$ increase in the odds of screen-detected type 2 diabetes. Assuming $7 \%$ prevalence of undiagnosed type 2 diabetes in neighbourhoods with no outlets (based on our data) and approximately 200 residents in a $500 \mathrm{~m}$ radius, then we would expect approximately fourteen people in a $500 \mathrm{~m}$ radius to have undiagnosed type 2 diabetes. Assuming that the number of fast-food outlets is causally associated with type 2 diabetes then our results suggest that for every additional two outlets per approximately 200 residents or $500 \mathrm{~m}$ radius, we would expect to see one more diabetes case. We also noted in our data that type 2 diabetes prevalence was fairly steady at approximately $8 \%$ when there were fewer than four outlets, at which point it increased to approximately $11 \%$ (data not shown). However, our data sampling method means that we will not have captured all of the outlets and so we cannot suggest a suitable cap on the number of outlets per $500 \mathrm{~m}$ radius from these data. Clearly more work is needed before guidance can be put in place, but the present study highlights that public health consideration needs to be given when granting planning permission to new fast-food outlets. Some local planning authorities in England already have such measures in place, but the evidence base for the restrictions that they impose is limited ${ }^{(29)}$. Furthermore, fast-food outlets themselves could potentially contribute towards reducing this problem by introducing healthier choices to their menus. A recent survey found that, from 2010 to 2013, the proportion of main dishes with healthy Nutrient Profile Index scores increased from $46 \%$ to $54 \%$ in five of the largest fast-food chains in the $\mathrm{USA}^{(30)}$. This suggests that steps are being taken by fast-food outlets to address the problem, but further action is required.

The current research highlights areas where knowledge is currently lacking. First, it needs to be ascertained whether there is a causal association between the number of fastfood outlets and obesity-related outcomes. While many of Bradford Hill's criteria for causation ${ }^{(31)}$ are satisfied (e.g. consistency, plausibility and biological gradient), unanswered questions remain around temporality, which would require longitudinal studies. Second, the intervention effect of reducing or limiting the number of fast-food outlets in a neighbourhood should be explored. Finally, precise measurement of the number of fast-food outlets is required 
to provide evidence for the upper limit of fast-food outlets in relation to the health of the residents.

\section{Conclusion}

In conclusion, the present study suggests that an increased number of fast-food outlets in the neighbourhood is associated with an increased risk of screen-detected type 2 diabetes and of being classified as obese, suggesting that fast-food outlets might be a reasonable target for public health interventions. However, these analyses are from cross-sectional data and conclusions should be interpreted with caution, with further research required, in particular to establish causality.

\section{Acknowledgements}

Financial support: The present research was supported by the National Institute for Health Research Collaboration for Leadership in Applied Health Research and Care - East Midlands (NIHR CLAHRC - EM), the Leicester Clinical Trials Unit and the NIHR Leicester-Loughborough Diet, Lifestyle and Physical Activity Biomedical Research Unit, which is a partnership between University Hospitals of Leicester NHS Trust, Loughborough University and the University of Leicester. ADDITION-Leicester was funded for support and treatment costs by the National Health Service (NHS) Department of Health Support for Science and project grants. Let's Prevent Diabetes was funded by an NIHR Programme Grant. Walking Away from Diabetes was supported by funding from the NIHR CLAHRC for Leicestershire, Northamptonshire and Rutland. The views expressed are those of the author(s) and not necessarily those of the NHS, the NIHR or the Department of Health. The study funders had no role in the design, analysis, or writing of this article. Conflict of interest: None. Authorship: K.K., M.J.D., D.W. and L.J.G. designed and conducted the ADDITIONLeicester study. K.K., M.J.D., L.J.G., T.Y. and S.H. designed and conducted the Let's Prevent Diabetes study. K.K., M.J. D., T.Y., C.E. and L.J.G. designed and conducted the Walking Away from Diabetes study. D.H.B., P.C., A.C. and K.K. conceived and designed the current analyses. D.H.B. performed the analyses and wrote the first draft of the manuscript with P.C. All authors contributed to interpreting the data, revising the manuscript and approved the final version. D.H.B. is the guarantor of the study. Ethics of buman subject participation: Ethical approval was from the University Hospitals of Leicester and Leicestershire Primary Care Research Alliance (ADDITION-Leicester) and the Nottingham (Walking Away from Diabetes/Let's Prevent Diabetes) Research Ethics Committees.

\section{Supplementary material}

To view supplementary material for this article, please visit http://dx.doi.org/10.1017/S1368980014002316

\section{References}

1. Whiting DR, Guariguata L, Weil C et al. (2011) IDF Diabetes Atlas: global estimates of the prevalence of diabetes for 2011 and 2030. Diabetes Res Clin Pract 94, 311-321.

2. Guh DP, Zhang W, Bansback N et al. (2009) The incidence of co-morbidities related to obesity and overweight: a systematic review and meta-analysis. BMC Public Health 9, 88 .

3. Pereira MA, Kartashov AI, Ebbeling CB et al. (2005) Fastfood habits, weight gain, and insulin resistance (the CARDIA study): 15-year prospective analysis. Lancet $\mathbf{3 6 5}$, 36-42.

4. Khunti K, Gray LJ, Skinner T et al. (2012) Effectiveness of a diabetes education and self management programme (DESMOND) for people with newly diagnosed type 2 diabetes mellitus: three year follow-up of a cluster randomised controlled trial in primary care. BMJ 344, e2333.

5. National Institute for Health and Clinical Excellence (2011) Preventing Type 2 Diabetes: Population and Communitylevel Interventions. NICE Public Health Guidance no. 35. London: NICE.

6. Smith KJ, McNaughton SA, Gall SL et al. (2009) Takeaway food consumption and its associations with diet quality and abdominal obesity: a cross-sectional study of young adults. Int J Behav Nutr Phys Act 6, 29.

7. Moore LV, Roux AD, Nettleton JA et al. (2009) Fast-food consumption, diet quality, and neighborhood exposure to fast food. Am J Epidemiol 170, 29-36.

8. Duffey KJ, Gordon-Larsen P, Steffen LM et al. (2009) Regular consumption from fast food establishments relative to other restaurants is differentially associated with metabolic outcomes in young adults. $J$ Nutr 139, 2113-2118.

9. Astrup A, Dyerberg J, Selleck M et al. (2008) Nutrition transition and its relationship to the development of obesity and related chronic diseases. Obes Rev 9, 48-52.

10. Maddock J (2004) The relationship between obesity and the prevalence of fast food restaurants: state-level analysis. $\mathrm{Am} \mathrm{J}$ Health Promot 19, 137-143.

11. Jeffery RW, Baxter J, McGuire M et al. (2006) Are fast food restaurants an environmental risk factor for obesity? Int $J$ Behav Nutr Phys Act 3, 2.

12. Simmons D, McKenzie A, Eaton S et al. (2005) Choice and availability of takeaway and restaurant food is not related to the prevalence of adult obesity in rural communities in Australia. Int J Obes (Lond) 29, 703-710.

13. Holsten JE (2008) Obesity and the community food environment: a systematic review. Public Health Nutr 12, 397-405.

14. Webb DR, Khunti K, Srinivasan B et al. (2010) Rationale and design of the ADDITION-Leicester study, a systematic screening programme and randomised controlled trial of multi-factorial cardiovascular risk intervention in people with type 2 diabetes mellitus detected by screening. Trials 11, 16.

15. Gray LG, Khunti K, Williams S et al. (2012) Let's Prevent Diabetes: study protocol for a cluster randomised controlled trial of an educational intervention in a multiethnic UK population with screen detected impaired glucose regulation. Cardiovasc Diabetol 11, 56.

16. Yates T, Davies MJ, Henson J et al. (2012) Walking away from type 2 diabetes: trial protocol of a cluster randomized controlled trial evaluating a structured education programme in those at high risk of developing type 2 diabetes. BMC Fam Pract 13, 46.

17. Gray LJ, Khunti K, Edwardson C et al. (2012) Implementation of the automated Leicester Practice Risk Score in two diabetes prevention trials provides a high yield of people with abnormal glucose tolerance. Diabetologia 55, 3238-3244. 
18. World Health Organization (2011) Use of Glycated Haemoglobin (HbA1c) in the Diagnosis of Diabetes Mellitus. Geneva: WHO.

19. WHO Expert Consultation (2004) Appropriate body-mass index for Asian populations and its implications for policy and intervention strategies. Lancet 363, 157-163.

20. Kaczynski AT, Johnson AJ \& Saelens BE (2010) Neighborhood land use diversity and physical activity in adjacent parks. Health Place 16, 413-415.

21. Leslie E, Cerin E \& Kremer P (2010) Perceived neighborhood environment and park use as mediators of the effect of area socio-economic status on walking behaviors. $J$ Phys Act Health 7, 802-810.

22. Sugiyama T, Thompson CW \& Alves S (2009) Associations between neighborhood open space attributes and quality of life for older people in Britain. Environ Behav 41, 3-21.

23. IPAQ Group (2005) Guidelines for Data Processing and Analysis of the International Physical Activity Questionnaire (IPAQ) - Short and Long Forms. http://www.ipaq.ki.se/ scoring.pdf (accessed January 2014).

24. Fleischhacker SE, Evenson KR, Rodriguez DA et al. (2011) A systematic review of fast food access studies. Obes Rev 12, e460-e471.
25. Jaworowska A, Blackham T, Davies IG et al. (2013) Nutritional challenges and health implications of takeaway and fast food. Nutr Rev 71, 310-318.

26. Prentice AM \& Jebb SA (2003) Fast foods, energy density and obesity: a possible mechanistic link. Obes Rev 4, 187-194.

27. Buck D \& Frosini F (2012) Clustering of Unhealthy Behaviours over Time: Implications for Policy and Practice. London: The King's Fund.

28. Gholap N, Davies M, Patel K et al. (2011) Type 2 diabetes and cardiovascular disease in South Asians. Prim Care Diab 5, $45-56$.

29. Ross A (2013) Obesity-based policies to restrict hot food takeaways: progress by local planning authorities in England. http://www.medway.gov.uk/pdf/Obesity-based\% 20policies\%20to\%20restrict $\% 20$ hot $\% 20$ food\%20takeaways- $\% 20$ progress\%20by\%20local\%20planning\%20authorities\%20in\% 20England.pdf (accessed February 2014).

30. Harris JL, Schwartz MB, Munsell CR et al. (2013) Fast Food FACTS 2013: Measuring Progress in Nutrition and Marketing to Children and Teens. New Haven, CT: Yale Rudd Center for Food Policy \& Obesity.

31. Bradford Hill A (1965) The environment and disease: association or causation? Proc R Soc Med 58, 295-300. 